\title{
Inhibition of thioredoxin A1 from Corynebacterium pseudotuberculosis by polyanions and flavonoids
}

\author{
Raphael J. Eberle ${ }^{\mathrm{a}}$, Liege A. Kawai ${ }^{\mathrm{a}}$, Fabio R. de Moraes ${ }^{\mathrm{a}}$, Danilo Olivier ${ }^{\mathrm{a}}$, Marcos S. do Amaral ${ }^{\mathrm{b}}$, Ljubica Tasic ${ }^{\mathrm{c}}$, \\ Raghuvir K. Arni ${ }^{\text {a,* }}$, Monika A. Coronado ${ }^{\text {a,* }}$

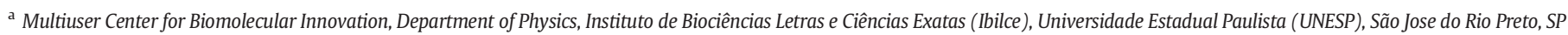 \\ 15054-000, Brazil \\ ${ }^{\mathrm{b}}$ Institute of Physics, Federal University of Mato Grosso do Sul, Campo Grande, MS 79090-700, Brazil \\ c Chemical Biology Laboratory, Organic Chemistry Department, Institute of Chemistry, University of Campinas (UNICAMP), Campinas, SP 13083-970, Brazil
}

\section{A R T I C L E I N F O}

\section{Article history:}

Received 5 February 2018

Received in revised form 4 June 2018

Accepted 5 June 2018

Available online 7 June 2018

\section{Keywords:}

Corynebacterium pseudotuberculosis

Thioredoxin

NMR

Inhibitor

Molecular dynamics

\begin{abstract}
A B S T R A C T
In pathogens, the thioredoxin system forms part of the defense against oxidative stress and ensures the formation of the proper disulfide bonds to ensure protein function. In Corynebacterium pseudotuberculosis, the role and mechanism of TrxA1 has not been elucidated, but, the significant homology among different Trxs and the conservation of the residues that form their active sites underline the importance of the Trx systems. Proteins involved in redox metabolism and low molecular weight thiols, which might interact with them, become attractive targets to modulate the activity of pathogens.

The activity of the protein was investigated using a turbidimetric assay system. The influence of different $\mathrm{pH}$ and low molecular weight thiols were tested. Additionally, this assay was used to investigate the inhibitory potential of ligands from different molecular families, such as, polyanions (suramin and heparin) and flavonoids (hesperetin and hesperidin). All four compounds showed inhibition of the protein activity by approximately 80\%. The interactions between these compounds and $C p$-TrxA1 were investigated using CD spectroscopy, NMR, molecular docking and dynamics. Our results demonstrate that suramin and hesperetin can serve as lead molecules for the development of specific inhibitors for the C. pseudotuberculosis TrxA1.
\end{abstract}

(C) 2018 Elsevier B.V. All rights reserved.

\section{Introduction}

Corynebacterium pseudotuberculosis (C. pseudotuberculosis) together with the Mycobacterium, Nocardia, and Rhodococcus genus constitutes the heterogeneous CMNR-group of pathogens [1]. C. pseudotuberculosis is the causative agent of caseous lymphadenitis (CLA), a disease encountered in sheep, goats, equids (ulcerative lymphangitis), and cattle (cutaneous excoriated granulomas). CLA infection results in significant reductions in wool and milk yields, causes cattle weight loss, leads to carcass condemnation and, can eventually, lead to death thereby

Abbreviations: CLA, Caseous lymphadenitis; CD, circular dichroism; CoASH, coenzymeA; DTT, dithiothreitol; ESH, ergothioneine; GSH, glutathione; Hst, hesperetin; Hsp, hesperidin; LMW thiol, low-molecular weight thiol; Trx, thioredoxin; SEC, size exclusion column; MD, molecular dynamics; CpHMD, constant pH-titration molecular dynamics; RMSF, root mean square fluctuation; RMSD, root mean square deviation; MM/GBSA, molecular mechanics/generalized Born surface area; NVT, constant volume and temperature; NPT, constant pressure and temperature; PME, particle-mesh Ewald method; STD-NMR, saturation transfer difference-nuclear magnetic resonance.

* Corresponding authors.

E-mail addresses: fabiom@ibilce.unesp.br, (F.R. de Moraes), marcos.amaral@ufms.br, (M.S. do Amaral), ljubica@iqm.unicamp.br, (L. Tasic), arni@sjrp.unesp.br, (R.K. Arni), monikacoronado@gmail.com (M.A.Coronado). resulting in considerable economic loss [2,3] and isolated cases of infection have also been reported in humans [4]. As a facultative intracellular parasite, this bacterium is capable of survival and growth in macrophages and is thus able to evade detection by the host immune system [5].

Redox metabolism represents an alternative, attractive target to control and/or modulate rapidly growing pathogens such as $C$. pseudotuberculosis. Low molecular weight (LMW) thiols play key roles in ensuring and modulating a reducing intracellular environment which is essential for the maintenance of regular metabolic activities [6, 7]. Besides the LMW thiols, many organisms contain thiol-disulfide oxidoreductases such as thioredoxins that function through their redox-active disulfides as reducing agents on protein disulfide bonds [8, 9].

Thioredoxins (Trx) are low molecular weight oxidoreductases $(\sim 12 \mathrm{kDa})$, structurally characterized by a central four-stranded distorted anti-parallel $\beta$-sheet surrounded by three $\alpha$-helices $[10,11]$. In vitro experiments indicate that the disulfide bonds in several proteins are efficiently reduced by thioredoxin $[9,12,13]$. Upon the reduction of the disulfide bonds, Trxs are oxidized and are subsequently reduced by thioredoxin reductase (TrxR) by utilizing NAD(P)H [9]. Trxs play crucial roles in the regulation of cellular redox homeostasis in pathogens, for 
example, $M$. tuberculosis relies on the Trx redox system for survival in conditions of elevated environmental stress [14]. M. tuberculosis and C. pseudotuberculosis are members of the CMNR-group and the actinomycetes and the Trx systems are essential for the survival of both pathogens.

We investigated the inhibitory potential of representative members from two distinct molecular classes i.e.; two polyanions (heparin, suramin) and two flavonoids (hesperetin and hesperidin) against $C$. pseudotuberculosis thioredoxin A1 (Cp-TrxA1). Polyanions are macromolecules and macromolecular complexes with multiple negative charges, and among others, are represented by proteoglycans (heparin), DNA, RNA, actin (microfilaments), tubulin (microtubules), polysialic acids, ribosomes, suramin, etc. [15].

In contrast, flavonoids belong to a large group of mildly charged phenolic compounds that are widely distributed in plants. Several biological properties have been reported for flavonoids, including antioxidant, anticancer, cancer chemo preventive, and anti-inflammatory properties $[16,17]$. Hesperidin (Hsd) is a flavanone glycoside (consisting of the disaccharide rutinose), which is abundant in citrus fruits. Its aglycone form is hesperetin (Hst) and both molecules are hydrophobic [18, 19].

Our study describes the production, purification and characterization of $C p$-TrxA1. Suramin, heparin, Hst and Hsp inhibited (>80\%) $C p$ TrxA1 and were evaluated using activity assay. The evaluation of the binding modes between polyanions and flavonoids by $\mathrm{CD}$ spectroscopy, NMR techniques and molecular dynamics indicated significant differences which are relevant for the development of specific inhibitors against this family of proteins.

\section{Material and methods}

\subsection{Chemical and biochemical reagents}

Kanamycin, chloramphenicol, $\mathrm{MgSO}_{4}$, IPTG, $\mathrm{K}_{2} \mathrm{HPO}_{4}, \mathrm{KH}_{2} \mathrm{PO}_{4}, \mathrm{NaCl}$, glycerol, imidazole, DTT, diamide, glutathione, coenzyme-A, ergothioneine, low molecular weight heparin (MW: $6000 \mathrm{kDa}$ ), suramin, hesperetin, EDTA, methanol, $\mathrm{D}_{2} \mathrm{O}$, 4,4-dimethyl-4silapentane-1-sulfonic acid, DMSO, acetic acid and isopropanol were purchased from Sigma-Aldrich (USA). Human insulin was purchased from Eli Lilly (Brazil). Hesperidin was produced and purified as described in Section 2.7.

\subsection{Overexpression and purification of $C p-\operatorname{Tr} x A 1$}

The E.coli BL21-CodonPlus (DE3)-RIL strain harboring the pD441-SR (DNA 2.0-USA) plasmid was cultured overnight in Luria-Bertani (LB) medium supplemented with sufficient amounts of kanamycin and chloramphenicol at $37^{\circ} \mathrm{C}$. The bacterial cultures were then inoculated into fresh LB medium (with the same antibiotic), till the final $A_{600}$ reached 0.6. At that point, $C p$-TrxA1 was supplemented with 50 $\mu \mathrm{mol} \mathrm{L}{ }^{-1} \mathrm{MgSO}_{4}$ and induced with $0.35 \mathrm{mmol} \mathrm{L}^{-1}$ IPTG, the incubation continued for $5 \mathrm{~h}$ at $30^{\circ} \mathrm{C}$. The cultures were harvested and the cell pellet re-suspended in $20 \mathrm{mmol} \mathrm{L} \mathrm{L}^{-1} \mathrm{~K}_{2} \mathrm{HPO}_{4} / \mathrm{KH}_{2} \mathrm{PO}_{4}, \mathrm{pH} \quad 7.4$, $500 \mathrm{mmol} \mathrm{L}^{-1} \mathrm{NaCl}, 5 \%(\mathrm{v} / \mathrm{v}$ ) glycerol. The cells were lysed by sonication in four sets of $30 \mathrm{~s}$ pulses of $30 \%$ amplitude, with $10 \mathrm{~s}$ intervals, followed by centrifugation at $8000 \times \mathrm{g}, 6^{\circ} \mathrm{C}$ for $90 \mathrm{~min}$. The supernatant containing the recombinant $\mathrm{Cp}$-TrxA1 with $6 \mathrm{x}$-His-tag was loaded onto a NiNTA column pre-equilibrated with the same buffer. To remove the unbound protein, the column was extensively washed with $20 \mathrm{mmol} \mathrm{L}^{-1}$ $\mathrm{K}_{2} \mathrm{HPO}_{4} / \mathrm{KH}_{2} \mathrm{PO}_{4}$, pH 7.4, 500 mmol L ${ }^{-1} \mathrm{NaCl}, 5 \%$ (v/v) glycerol, containing 20 and $60 \mathrm{mmol} \mathrm{L}^{-1}$ imidazole. Cp-TrxA1 was eluted with $500 \mathrm{mmol} \mathrm{L}^{-1}$ imizole. Eluted fractions were individually pooled, and injected onto a Superdex 75 10/300 GL (GE Healthcare) size exclusion column (SEC), pre-equilibrated with $20 \mathrm{mmol} \mathrm{L}{ }^{-1} \mathrm{~K}_{2} \mathrm{HPO}_{4} / \mathrm{KH}_{2} \mathrm{PO}_{4}$ $\mathrm{pH} 7.4,150 \mathrm{mmol} \mathrm{L}^{-1} \mathrm{NaCl}$. Sample purities, were assessed by SDSPAGE (20\%) gels after each purification step. Protein concentrations were determined spectrophotometrically by applying the LambertBeer law [20].

\subsection{Circular dichroism spectroscopy (CD)}

For CD measurements, 15 repeated scans were performed, of which 5 were used to establish the baseline. The wavelength range used to obtain the far-UV spectra was from $200 \mathrm{~nm}$ to $260 \mathrm{~nm}$, in a constant time of $1 \mathrm{~s}$ and $100 \mathrm{~nm} / \mathrm{min}$ continuous scanning mode, using a Jasco J-107 spectropolarimeter (Jasco, Japan). Cp-TrxA1 was diluted in $20 \mathrm{mmol} \mathrm{L}{ }^{-1} \mathrm{~K}_{2} \mathrm{HPO}_{4} / \mathrm{KH}_{2} \mathrm{PO}_{4}$. $20 \mathrm{mmol} \mathrm{L}{ }^{-1} \mathrm{~K}_{2} \mathrm{HPO}_{4} / \mathrm{KH}_{2} \mathrm{PO}_{4}$ at $\mathrm{pH} 7.4$ was used to determine the effect of $1.4 \mu \mathrm{mol} \mathrm{L} \mathrm{L}^{-1}$ : DTT, diamide, $\mathrm{GSH}, \mathrm{CoASH}, \mathrm{ESH}$, on the protein secondary structure. The effect of the ligands heparin, suramin, hesperidin (Hsd) and hesperetin (Hsp) were individually tested with $10 \mu \mathrm{mol} \mathrm{L}{ }^{-1}$ of $\mathrm{Cp}$-TrxA1. The protein was incubated with a fourfold molar excess of the ligands $\left(40 \mu \mathrm{mol} \mathrm{L}{ }^{-1}\right)$ at $4{ }^{\circ} \mathrm{C}$ for $2 \mathrm{~h}$. Subsequently, the protein was extensively dialyzed against $20 \mathrm{mmol} \mathrm{L}-1 \mathrm{~K}_{2} \mathrm{HPO}_{4} / \mathrm{KH}_{2} \mathrm{PO}_{4}$ to remove unbound ligand molecules and the results were analyzed using the CDpro software package [21].

\subsection{Nuclear magnetic resonance (NMR)}

\subsubsection{Saturation transfer difference by nuclear magnetic resonance (STD- NMR)}

Nuclear Magnetic Resonance (NMR) spectroscopy was used to identify the $C p$-TrxA1 interaction with potential modulators. All experiments were performed utilizing a Bruker AVANCE III HD spectrometer (Bruker, Germany) operating at $600 \mathrm{MHz}$ for ${ }^{1} \mathrm{H}$, equipped with a triple-resonance, pulsed-field, z-gradient cryoprobe. An off-resonance at $20 \mathrm{ppm}$ was chosen, and the on-resonance frequencies were selected according to the ligand signals. The Bruker pulse sequence STDDIFFESGP was used for the STD-NMR spectroscopy experiments. The protein was saturated for $2 \mathrm{~s}$, the recycle delay was set to $3 \mathrm{~s}$ and a saturation power of $35 \mathrm{dBW}$ was used. The protein signal was suppressed by utilizing a spin-lock filter of $30 \mathrm{~ms}$.

Each experiment used a solution of $400 \mu \mathrm{mol} \mathrm{L}{ }^{-1}$ of each ligand (heparin, suramin, Hsp, and Hst) with $20 \mu \mathrm{mol} \mathrm{L}^{-1}$ of $C p$-TrxA1 diluted in $20 \mathrm{mmol} \mathrm{L}^{-1} \mathrm{~K}_{2} \mathrm{HPO}_{4} / \mathrm{KH}_{2} \mathrm{PO}_{4}$, pH 7.4 and possible interactions were evaluated based on the STD-NMR results.

\subsubsection{Chemical shift mapping by nuclear magnetic resonance}

For protein-ligand identification and quantification, the protein chemical shifts which are usually sensitive to ligand binding were identified by ${ }^{15} \mathrm{~N}-\mathrm{HSQC}$ (Heteronuclear Single Quantum Coherence) experiments [22]. Since the NMR spectra have been assigned, binding sites could be identified by mapping them on the protein molecular surface. Chemical shift mapping using the ${ }^{1} \mathrm{H}$ NMR protein spectrum enabled the identification of shifts of tryptophan side chain indole groups upon ligand binding.

A Bruker Advance (Bruker, Germany) $600 \mathrm{MHz}$ equipped with a $5 \mathrm{~mm}$ triple resonance cryoprobe with pulse-field gradient along the $\mathrm{z}$-axis was used and for the regular excitation sculpting experiment (ZGESGP). Data was acquired for $C p$-TrxA1 in $20 \mathrm{mmol} \mathrm{L}^{-1} \mathrm{~K}_{2} \mathrm{HPO}_{4} /$ $\mathrm{KH}_{2} \mathrm{PO}_{4}$, pH 7.4 in $10 \% \mathrm{D}_{2} \mathrm{O}$ both in the presence and absence of each ligand. A temperature of $20^{\circ} \mathrm{C}$ was set and 128 transients were collected, with a slide window of $22 \mathrm{ppm}$ and acquisition time of $1.21 \mathrm{~s}$. Each spectrum was referenced to DSS, presented in $25 \mu \mathrm{mol} \mathrm{\textrm {L } ^ { - 1 }}$ in the solution. For $C p$-TrxA1-suramin interaction, a $0.9 \mathrm{mmol} \mathrm{L}^{-1}$ protein concentration was used and its spectrum was compared to one using $1.8 \mathrm{mmol} \mathrm{L}^{-1}$ ligand. For the flavonoid hesperitin, due to the hydrophobic nature, a $0.1 \mathrm{mmol} \mathrm{L}^{-1}$ protein sample was used and compared to the spectrum in the presence of $0.2 \mathrm{mmol} \mathrm{L}^{-1}$ of the ligand. Experiments were processed in Bruker TopSpin 3.2, applying a line broadening of $0.3 \mathrm{~Hz}$ prior to Fourier transformation. 


\subsection{Turbidimetric assay of insulin disulfide reduction}

The rate of insulin disulfide reduction by $C p$-TrxA1 was monitored spectrophotometrically following evaluation of the turbidity at $640 \mathrm{~nm}$ [23-26], using a Biomate UV-Visible Spectrophotometer (Thermo Fisher Scientific, USA). The reaction mixture contained a total volume of $500 \mu \mathrm{L}$, with $0.2 \mathrm{mmol} \mathrm{L}^{-1}$ insulin, $50 \mathrm{mmol} \mathrm{L}^{-1} \mathrm{Tris} / \mathrm{HCl}$, $2 \mathrm{mmol} \mathrm{L}^{-1}$ EDTA, and $5 \mu \mathrm{mol} \mathrm{L}{ }^{-1}$ protein. The optimum $\mathrm{pH}$ for the Cp-TrxA1 activity was determined by using Tris/HCl buffers at different pH values $(7.0,7.4,7.8,8.0,8.4,8.8$ and 9.0). The reactions occurred at 25 ${ }^{\circ} \mathrm{C}$ by adding $1 \mathrm{mmol} \mathrm{L}^{-1} \mathrm{DTT}$. In another experiment, instead of DTT, the reductive agents GSH, ESH, CoASH and the oxidant diamide, were used at a final concentration of $1 \mathrm{mmol} \mathrm{L}^{-1}$. As a control, the nonenzymatic reduction of insulin by the corresponding reductive agent was used.

Cp-TrxA1 activity was assayed in the presence of the following ligands: heparin, suramin, hesperidin, and hesperetin, all at final concentrations of $0.14 \mu \mathrm{mol} \mathrm{L}^{-1}$. The protein was incubated with the ligands overnight at $4{ }^{\circ} \mathrm{C}$ and, subsequently, the samples were injected onto a Superdex 75 10/300 GL (GE Healthcare) column pre-equilibrated with $20 \mathrm{mmol} \mathrm{L}^{-1} \mathrm{Tris} / \mathrm{HCl} \mathrm{pH} 7.4,150 \mathrm{mmol} \mathrm{L}^{-1} \mathrm{NaCl}$, to remove nonspecifically bound ligands, and then tested using the reaction mixture described above. The experiments were performed as triplet measurements and repeated twice.

\subsection{Molecular dynamics and computational analysis of Cp-TrxA1}

\subsubsection{Starting structures and molecular docking}

The initial model of the $C p$-TrxA1 protein was obtained by homology modeling with the M. avium Trx structure (PDB: 4WXT; 50\% homology), in order to obtain a refined and relaxed model, the protein was solvated and ions were added to neutralize the system. Following which, 100 ns Molecular Dynamics (MD) simulations were carried out and clustering analysis were performed to obtain a stable and representative model (Fig. S1A, Supplementary material). Structural superposition of the $C p$-TrxA1 homology model and the $M$. avium Trx structure showed a RMSD of 0.188 A (Fig. S1B, Supplementary material).

The model was used as a starting conformation for constant $\mathrm{pH}$ MD (CpHMD), for docking calculations and posterior MD simulations with ligands. To prepare the ligands, Gaussian 09 [27] was used at the level of theory HF/6-31G* to optimize and calculate their ESP (Electrostatic Surface Potential). Following which, the antechamber program [28] was used to calculate their RESP (Restrained Electrostatic Potential) charges and parmchk program to obtain the missing GAFF (General Amber Force Field) parameters [29].

Docking calculations were performed using AutoDock Vina 1.1.12 [30]. The AutoDockTools program [31] was used adding polar hydrogens and partial charges to the protein and set the rotational bonds in the ligands. The search space was defined in the gridbox near the binding site. As output results, for each calculation, several poses were ranked according to the scoring function of AutoDock Vina. Thus, the first poses for $C p$-TrxA1-Hst and $C p$-TrxA1-suramin complexes were used to initiate MD simulations.

\subsubsection{General setup and parameterization of the molecular dynamics simulations}

All simulations were carried out using the AMBER16 [32] program package. The FF14SB force field [33] was used to describe the all-atom protein interaction, whereas the GAFF and RESP charges were used to describe the ligands. The $\mathrm{H}++$ web-server [34] was used to set the amino acid protonation state at $\mathrm{pH}$ 7.0. The systems were neutralized with $\mathrm{Na}^{+}$ions and placed in a rectangular box of TIP3P water extended $10 \AA ̊$ away from any protein atom. To exclude bad contacts from initial structures, each system was minimized in two steps. First, the energy minimization of the protein-ligand constrained complexes (force constant of $50.0 \mathrm{kcal} / \mathrm{mol}-\AA^{2}$ ) were performed with 2500 steepest descent steps followed by 2500 conjugate gradient steps followed by unconstrained energy minimization rounds (10,000 steps). After minimization, the system was gradually heated from 0 to $293 \mathrm{~K}$ for 300 ps under NVT ensemble (constant volume and temperature), while the protein was restrained with force constant of $25 \mathrm{kcal} / \mathrm{mol}$ $\AA^{2}$. Subsequently, an equilibration step was performed using NpT ensemble (constant pressure and temperature) for 500 ps. Finally, the production runs, 100 and 150 ns for $C p$-TrxA1-Hst and Cp-TrxA1-suramin, respectively, were performed in NpT ensemble without any restraints. The temperature (293 K) and pressure (1 atm) were controlled by Langevin coupling. The SHAKE constraints were applied to all bonds involving hydrogen atoms to allow a 2-fs dynamics time step. Long-range electrostatic interactions were calculated by the particle-mesh Ewald method (PME) using 10-Å cutoff.

\subsubsection{Constant $\mathrm{pH}$-titration $\mathrm{MD}$}

To understand how $\mathrm{pH}$ affect the protein dynamics, we used the CpHMD. First, to prepare the system, the names of the residues Glu and Asp were changed, respectively, to GL4 and AS4 to allow the correct treatment by AMBER. During the simulation, all the GL4, AS4, LYS and CYS residues were titrated, while the rest of residues existed in a fixed protonation state.

The CpHMD simulations were performed following a procedure that resembles a titration experiment in a wet-laboratory, as introduced by Socher and Sticht [35]. The idea is to divide the MD into a large number of small steps and change the $\mathrm{pH}$ after each step. In our setup, the MD started at $\mathrm{pH} 7$ and lasted for $1 \mathrm{~ns}$ in each step. After that, using the final coordinates and velocity of the previous MD as an input, we increased the solvent $\mathrm{pH}$ by $0.05 \mathrm{pH}$ unit and run another round. The $\mathrm{pH}$ range was from 7.0 to 9.0, generating a total of 140 short 1-ns MD simulations and a total time of $140 \mathrm{~ns}$. The protonation was checked every 10 steps, according to the MC (Monte Carlo) sampling of the Boltzmann distribution of protonation states. For the protonation check, it strips all the explicit solvent and uses the generalized Born solvent model (igb = 2). After protonation check the MD simulation could follow two directions: if the protonation changes, it ran 100 steps of MD simulation to allow the solvent relaxation around the new titratable site; or, if the protonation did not change, it restored the solvent and continued the MD simulation from the same point.

\subsubsection{Structural and dynamical analyses}

All the analyses were performed with the CPPTRAJ program [36] of AmberTools17. VMD and Pymol were used for visualization of the systems. RMSD of $\mathrm{C} \alpha$ was calculated to determine the equilibration and convergence of the systems. Clustering analysis was done with kmeans method ranging from 2 to 6 . To access the quality of clustering we use the DBI values and also silhouette analyses. Protein flexibility was studied by root mean square fluctuation (RMSF) for the C $\alpha$. The RMSF were calculated residue-by-residue over the equilibrated trajectories.

The interaction energy was calculated using the molecular mechanics/generalized Born surface area (MM/GBSA) approach. MM/GBSA energy was computed between the protein and the ligands in the stable regime comprising the last $10 \mathrm{~ns}$ of the MD simulation. For these calculations, all the solvent and ions were stripped.

The secondary structure content of the $C p$-TrxA1 homology model and the complexes with suramin and Hst after MD simulations were evaluated with PDBsum [37].

\subsection{Production and purification of hesperidin (Hsp)}

Hesperidin was obtained according to Tasic et al. [38]. 


\section{Results and discussion}

\subsection{Enzyme production and purification}

The recombinant expressed $C p$-TrxA1 was purified by affinity chromatography using a Ni-NTA column. The recombinant protein was eluted with $500 \mathrm{mmol} \mathrm{L}^{-1}$ imidazole; a subsequent purification step was performed using SEC to remove trace protein impurities. After SEC, a single protein band with a molecular mass of around $14 \mathrm{kDa}$ was observed on a $20 \%$ SDS-PAGE gel, the result correlated with the predicted molecular mass ( 13,5 kDa; with 6x-His-tag) (Fig. S2, Supplementary material).

\subsection{Enzyme activity and $\mathrm{pH}$ effect on the catalytically process of $\mathrm{Cp}$-TrxA1}

The $C p$-TrxA1 activity was tested by a turbidimetric insulin reduction assay. The catalytic effect of $C p$-TrxA1 resulted in the rapid precipitation of insulin as observed after $10 \mathrm{~min}$, and reached the saturation level after $38 \mathrm{~min}$. The non-enzymatic reduction of insulin by DTT, used as a control, started after $20 \mathrm{~min}$ and a lower precipitation level of insulin was observed (Fig. 1A).

The effects of the $\mathrm{pH}$ on the $C p$-TrxA1 activity were determined for the insulin reaction and, the results demonstrated that the optimum $\mathrm{pH}$ at room temperature was 7.4. Interestingly a second $\mathrm{pH}$ optimum was observed at $\mathrm{pH}$ 8.4. Constant $\mathrm{pH}$-titration molecular dynamics (CpHMD) were used to investigate the influence of the $\mathrm{pH}$ on the relevant amino acids for the protein activity, Cys32, Cys35 and, Trp31. As a parameter the solvent accessible surface $\left[\AA^{2}\right]$ of these residues were followed during the MD.

At pHs 7.4 and 8.4, the molecular surface is less accessible than at pHs 7.3 and 7.6, for both cysteines. Our results indicate that the libration of the Trp31 indole ring plays a role in modulating the accessibility of both cysteine ( 32 and 35) and may be involved in guiding the substrate (Fig. S3, Supplementary material).

MD simulations at $\mathrm{pH} 7.3$ and $\mathrm{pH} 7.6$ suggest that the position of the Trp31indole ring leads to formation of an 'open' active site Fig. S3. In this orientation, Trp31 stabilizes the interaction with the substrate [39] and, also forms hydrogen bonds with Cys32 [40]. The pH induced molecular movement of Trp31 can perturb this effect resulting in decreased protein activity as described above.

\subsection{Enzyme specificity and identification of potential inhibitors}

The experiments to evaluate the influence of redox agents (DTT, GSH, ESH, CoASH and diamide) and ligands (heparin, suramin, Hsd and Hst) on the enzymatic reduction of insulin by $C p$-TrxA1 were performed at $\mathrm{pH} 7.4$.

DTT, showed the strongest effect on the protein activity, followed by ESH and GSH. The other tested reduction agents (CoASH and diamide) presented reduced effects on the protein (Fig. 2A).

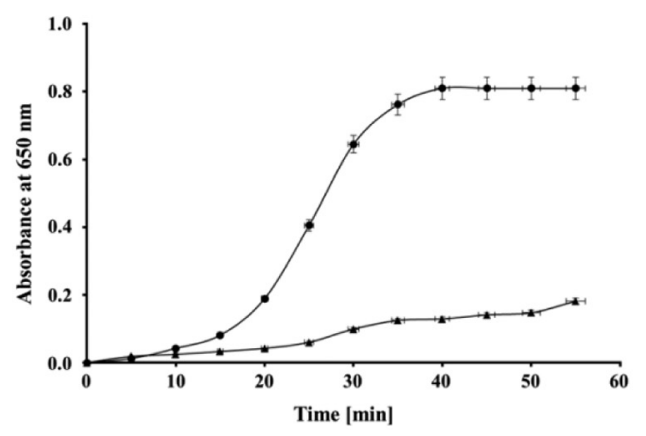

Besides, DTT, ESH produced the strongest effect on insulin reduction by $C p$-TrxA1, an event that has not been described previously. ESH is a 2-thiol-L-histidine betaine $[12,41]$ and possesses antioxidative activity [42].

The tripeptide GSH decreased activity of Cp-TrxA1 about 50\% when compared with DTT. GSH interacts preferably with Grx proteins and is not the preferred substrate of Trx proteins and, the ability of GSH to reduce $C p$-TrxA1 indicates the variability for substrate preference of $C p$ TrxA1.

The oxidation of the $C p$-TrxA1 thiol active site caused by diamide led to inhibition of the protein.

The polyanions heparin, suramin, and the two flavonoids, Hsp and Hst, where tested for their inhibitory potential against the insulin reducing capacity of $C p$-TrxA1. The molecular structures of these ligands are presented in Fig. S4. These molecules inhibit manifold proteins [43-48]; however, their interactions with Trx proteins have not been investigated, recently, their effects on the C. pseudotuberculosis Grx protein were described [49].

To eliminate the effects of unbound ligands on insulin, an additional gel filtration step was used. The insulin reduction activity of $C p$-TrxA1 was inhibited ( $>85 \%$ ) by all four tested ligands (Fig. 2B). The flavanone glycoside Hsp, and its aglycone form (lacking the disaccharide rutinose) Hst [37], possesses anticancer, cancer preventive, antiinflammatory, neuroprotective and antioxidant activities [45-47, 50].

Heparin is a linear, polydisperse polysaccharide, consisting of repeating units of $1 \rightarrow 4$-linked pyranosyluronic acid and 2-amino-2deoxyglucopyranose (glucosamine) residues [51]. The negatively charged sulfo or carboxyl groups of heparin interact with positively charged basic amino acids in the proteins [45]. A similar binding mode is observed for suramin, a symmetric divalent molecule possessing two naphthalene-trisulfonic acid heads, with negative charges. Suramin is a well-characterized analogue of heparin and both molecules compete for the same binding region in a number of proteins [44, 52, 53].

During the Trx activity the active site thiols need to be reduced and after this process they are oxidized, the formation of an intramolecular disulfide bridge includes changes in the secondary structure of the protein, visible using $C D$ spectroscopy.

Far-UV CD spectra of Cp-TrxA1 at pH 7.4 in phosphate buffer were used to investigate the changes of the secondary structure activated by DTT and GSH (Fig. 2C). When incubated with DTT or GSH, the secondary structure composition of $C p$-TrxA1 changed. When the protein was incubated with diamide, which oxidizes the thiol at the active site, the secondary structure composition is identical to the untreated $C p$-TrxA1. The secondary structural changes in the reduced and oxidized $C p$-TrxA1 homology model are presented in Fig. S5, Supplementary material. ESH and CoASH caused slight modifications of the $\mathrm{Cp}$ TrxA1 CD signals, which are divergent, to very significant changes induced by GSH and DTT. The results of the insulin reduction assay indicated a strong influence of ESH on the Cp-TrxA1 activity, but since the binding mode is unknown, further investigations are required. The

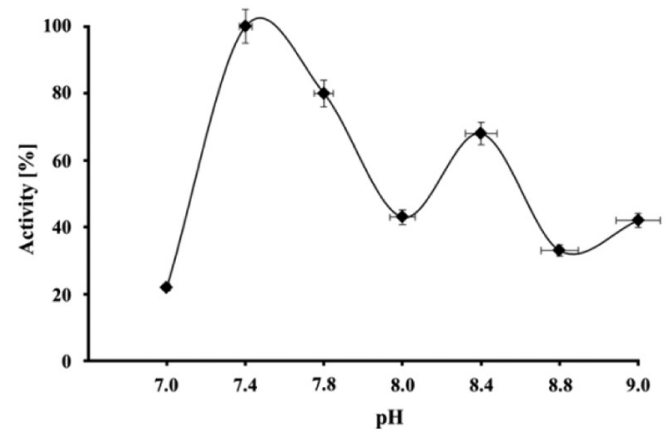

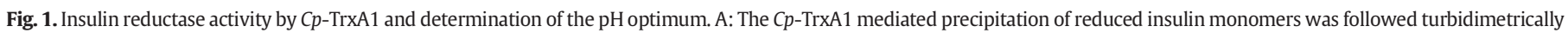
at $640 \mathrm{~nm}$; the non-enzymatic reduction of insulin by DTT served as the control. B: pH profile of Cp-TrxA1 in the pH range 7.0 to 9.0 using the insulin reductase assay. 
$\mathbf{A}$
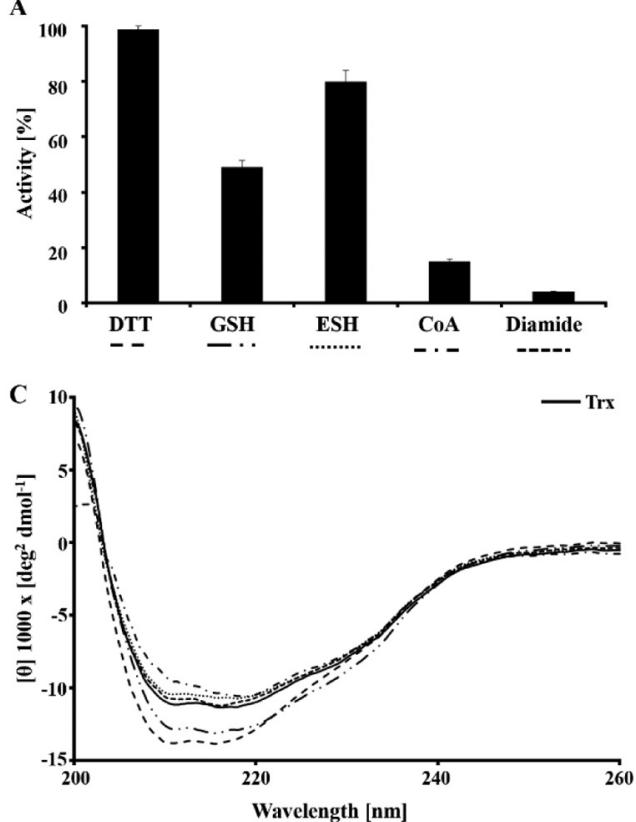

B
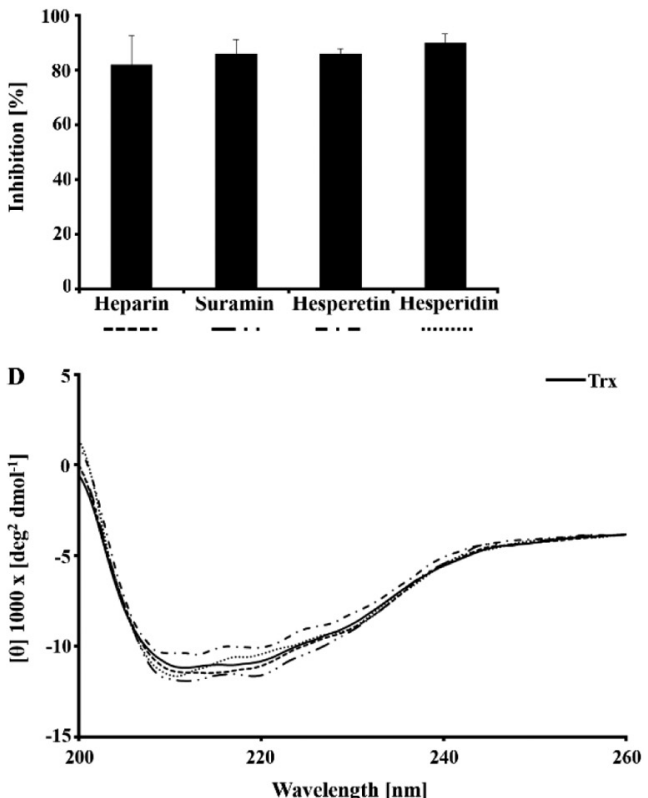

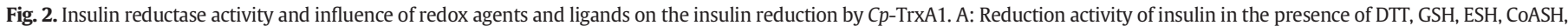

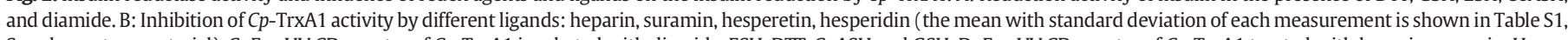

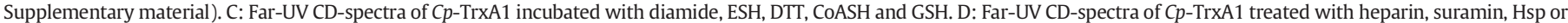
Hst (The CD spectroscopy results were evaluated by utilizing the CDpro software, Table S2, Supplementary material).

inhibitors identified in the insulin reduction assay, were investigated regarding their effect on the secondary structure of the proteins (Fig. 2D). The strongest influence on $C p$-TrxA1 was observed in the presence of Hst. It has been shown that the interactions of several Trx proteins with inhibitors or ligands result in conformational changes [14, 54, 55]. Suramin and heparin showed similar effects but not so strong like Hst. Interestingly the two tested molecule classes, flavonoids (Hst and Hsp) and polyanions (heparin and suramin) showed each a different effect on the protein secondary structure, which would assume a different mode of interaction.
3.4. Binding studies between Cp-TrxA1 and potential inhibitors using nuclear magnetic resonance (NMR) and MD simulations

STD-NMR experiments of $C p$-TrxA1 could confirm the interaction of the protein with suramin and Hst. However, experiments involving $C p$ TrxA1 with heparin and Hsp did not result in observable STD-NMR signals in the difference spectra. For Hsp interaction, it may be due to the affinity cut-off associated with STD-NMR experiments, which requires the ligand to dissociate from the protein receptor to enable successful measurement of the binding event. For heparin, its relatively high
A

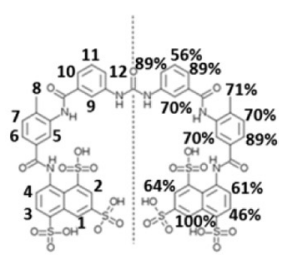

Suramin
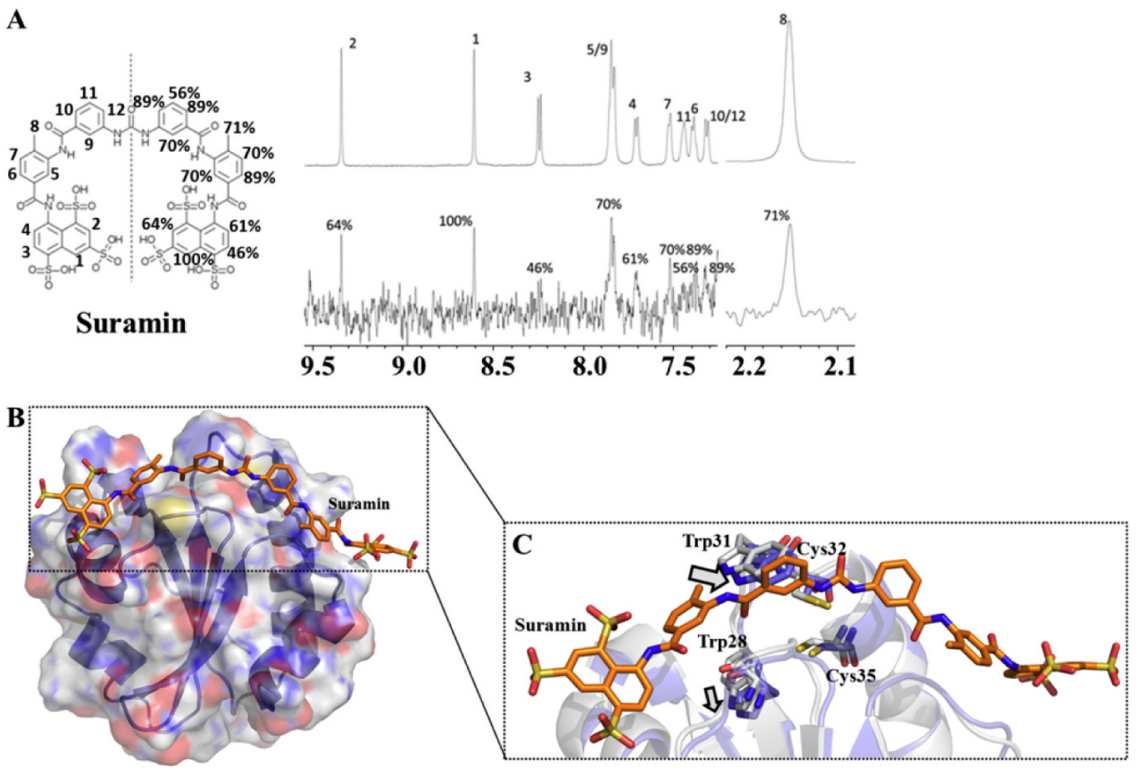

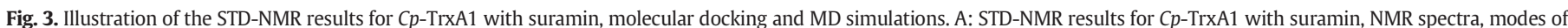

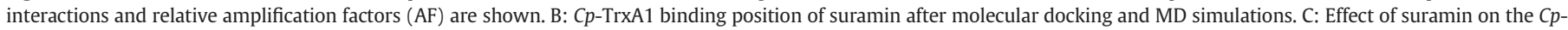

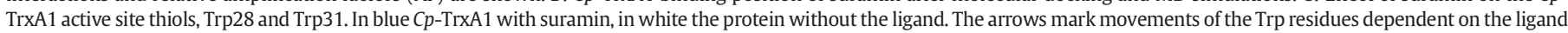
binding. 
molecular mass impairs STD from being effective. Protein-ligand complexes with dissociation constants lower than a few micro molars are not directly observed in STD-NMR experiments.

Suramin interacts predominantly with $C p$-TrxA1 via the sulfonic acid groups, as the highest relative STD-AF of $100 \%$ was observed for this moiety (Fig. 3A).

Molecular docking of suramin with $C p$-TrxA1 followed by MD was used to identify the region of interaction between suramin and the protein (Fig. 3B). The interaction energy calculated out of these experiments for the $C p$-TrxA1 and suramin complex was $-32.5 \pm 0.3 \mathrm{kcal} / \mathrm{mol}$. Interestingly, the results of molecular docking and MD simulations demonstrated that suramin interacts with the protein in the region which forms the binding surface between the protein and substrate or TrxR (Fig. S6A) [56-58]. Obviously, the interaction of suramin in this protein region interferes with substrate binding and demonstrates a possible mode of inhibition. Furthermore MD results have demonstrated the effect of suramin on Trp31 and the active site cysteine (Fig. 3C). Trp31 interacts thereby directly with suramin as demonstrated by the decomposition energy, which was determined for all residues (Fig. S7A).

Complementary NMR experiments indicated that the interaction with suramin changes the micro-environment of both Trp residues in the protein (Trp28 and Trp31). Indicating the binding of suramin at a nearby site, the tryptophan indole group was used to identify binding of suramin. The ligand is observed to promote significant chemical shift change in the area around 10 ppm (Fig. S8). As described earlier,
Trp31 is important for protein activity, the interaction of suramin with this residue can affect protein catalysis.

Evaluating the Hst binding epitopes is more challenging since hydrogen signals are superposed in the 1D spectra. However, the highest relative STD-AF of $100 \%$ is determined for the hydrogens of the aromatic ring system in the Hst molecule (Fig. 4A).

Molecular docking and MD simulations indicates the interaction of Hst with $C p$-TrxA1 near the protein active site (Fig. 4B), mainly with $\alpha$-helix three, thereby the calculated interaction energy was $-14.8 \pm$ $0.1 \mathrm{kcal} / \mathrm{mol}$. Compared with the energy calculated for the Cp-TrxA1suramin complex the value is around two times lower. Suramin is a strong negatively charged molecule with a net charge of -6 , Hst has a net charge of zero, these differences in the charges and the resulting ionic interactions with positively charged amino acids of the protein can explain the difference in the interaction strength.

Hst interacts with $\alpha$-helix, which is involved in the Trx protein substrate binding surface [56-58] (Fig. S6B). Moreover, compared with the suramin position, the Hst location is different and appears does not directly restrict access to the active site, but MD results indicated an effect on Trp31 (Fig. 4C), which is indicated additionally by the decomposition energy for the interaction between Trp31 and Hst (Fig. S7B). The effect on Cp-TrxA1 Trp28 and 31 could also be observed by additionally NMR experiments, which demonstrated the effect on the micro environment of the Trp residues similar as described before for the suramin interaction (Fig. S8).

A
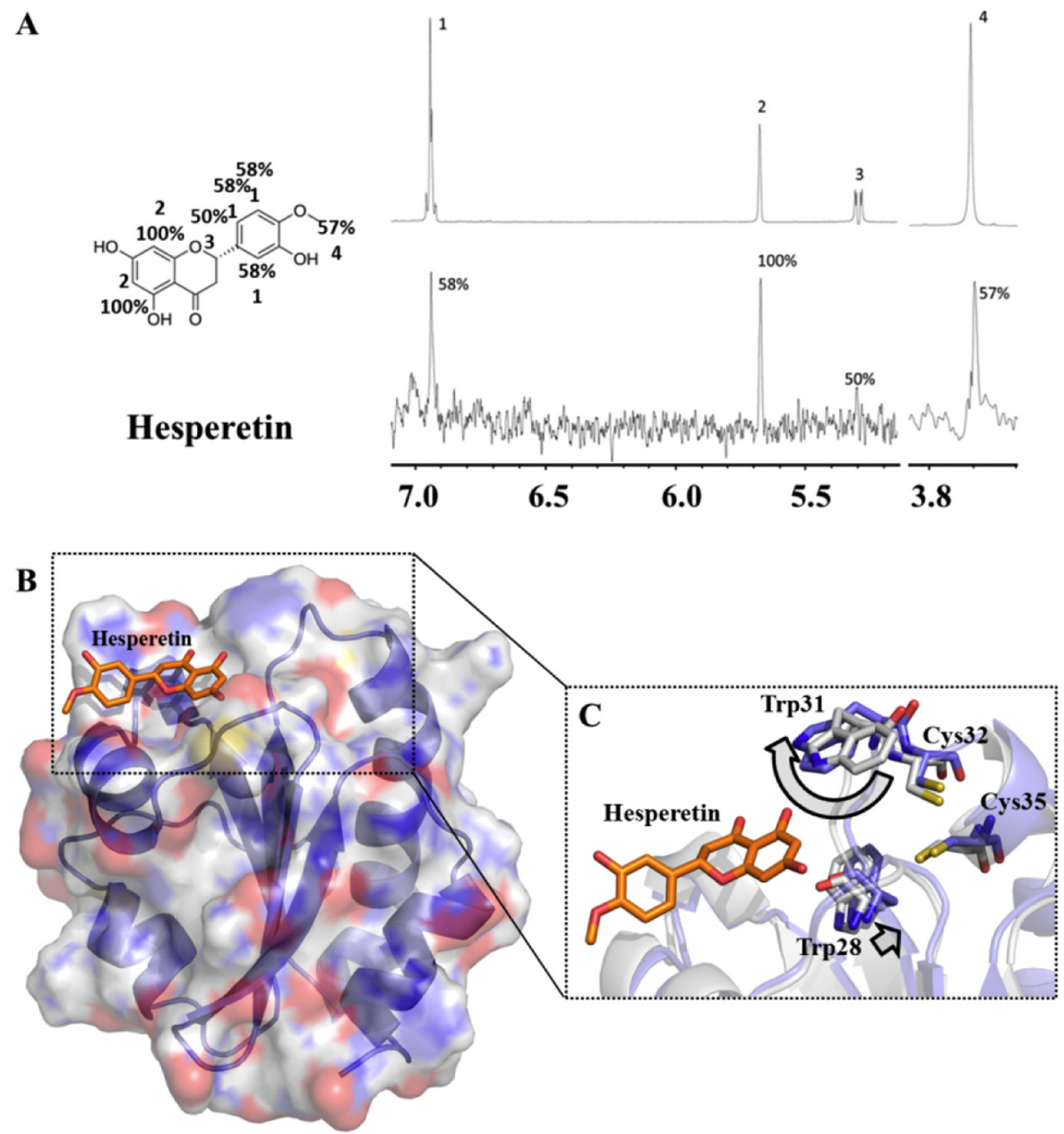

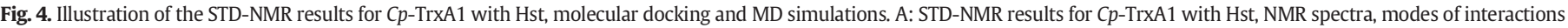

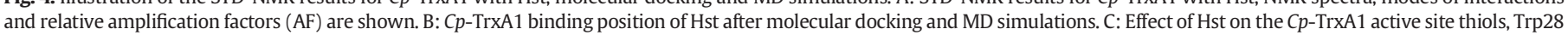
and Trp31. In blue $C p$-TrxA1 with Hst, in white the protein without the ligand. The arrows mark movements of the Trp residues dependent on the ligand binding. 
The binding of suramin and Hst with $C p$-TrxA1 influence molecular movements in the protein structure effecting the secondary structure content. It is demonstrated by MD simulations of the protein complex with the ligands and the $C D$ results as described before. The $\beta$-sheets content in the protein core remain unaffected, however, after ligand binding changes in the $\alpha$-helix content can be observed (Table S2, Supplementary material).

\section{Conclusion}

Trx proteins play crucial roles in the regulation of cellular redox homeostasis in pathogens, which is essential for their survival in situations of elevated environmental stress. The pathogens are in close contact with many molecules, which possess a strong influence on the cellular redox homeostasis. The high degree of conservation of the Trx active sites, and their similar three-dimensional folds indicate that inhibition of these proteins is likely to play an important role in pathogen viability.

The combination of activity assays, CD spectroscopy, NMR and MD simulations of the $\mathrm{Cp}$-TrxA1 Hst and suramin complexes provides interesting insights regarding these interactions. Both molecules inhibit protein activity by $>80 \%$, but the localizations and binding modes of these inhibitors are different. The inhibitors belong to different molecular classes; suramin is a polyanion whereas Hst is a flavonoid. Both molecules display significant differences in their electro physical behaviors. Our results demonstrate that polyanions and flavonoids can influence the activity of $C p$-TrxA1 and depending on the different binding modes as indicated by MD simulations, the Trx inhibitor does not interact directly with the active site cysteins. Restricting access to the substrate binding surface or Trp31, the active site plays a crucial role in determining the protein activity. These results can support the search and development of specific inhibitors for $C p$-TrxA1 as a means to combat $C$. pseudotuberculosis infections.

\section{Conflict of interest}

Authors declare that there exists no conflict of interest.

\section{Acknowledgements}

The authors want to thank Jorge Enrique Hernández from the physics department of the UNESP, Sao Jose do Rio Preto, Brazil, for providing the charges and force field parameters for suramin. This research was supported by grants from CNPq [Grant numbers 435913/2016-6, 401270/2014-9, 307338/2014-2, 150444/2017-6], FAPESP [Grant numbers 2015/13765-0, 2015/18868-2, 2016/08104-8; 2016/12904-0; 2009/53989-4], Fundect [23/200.307/2014], CAPES and PROPe UNESP.

\section{Appendix A. Supplementary data}

Supplementary data to this article can be found online at https://doi. org/10.1016/j.ijbiomac.2018.06.022.

\section{References}

[1] F.A. Dorella, L.G. Carvalho, S.C. Oliveira, A. Miyoshi, V. Azevedo, Corynebacterium pseudotuberculosis: microbiology, biochemical properties, pathogenesis and molecular studies of virulence, Vet. Res. 37 (2006) 201-218.

[2] M.W. Paton, I.R. Rose, R.A. Hart, S.S. Sutherland, A.R. Mercy, T.M. Ellis, J.A. Dhaliwal, New infection with Corynebacterium pseudotuberculosis reduces wool production, Aust. Vet. J. 71 (1994) 47-49.

[3] J.L. Ayers, Caseous lymphadenitis in goats and sheep: a review of diagnosis, pathogenesis, and immunity, J. Am. Vet. Med. Assoc. 171 (1977) 1251.

[4] M.M. Peel, G.G. Palmer, A.M. Stacpoole, T.G. Kerr, Human lymphadenitis due to Corynebacterium pseudotuberculosis: report of ten cases from Australia and review, Clin. Infect. Dis. 24 (1997) 185-191.

[5] S. Mckean, J. Davies, R. Moore, Identification of macrophage-induced genes of Corynebacterium pseudotuberculosis by differential fluorescence induction, Microbes Infect. 7 (2005) 1352-1363.
[6] A. Gaballa, G.L. Newton, H. Antelmann, D. Parsonage, H. Upton, M. Rawat, A Claiborne, R.C. Fahey, J.D. Helmann, Biosynthesis and functions of bacillithiol, a major low-molecular-weight thiol in Bacilli, Proc. Natl. Acad. Sci. U. S. A. 107 (2010) 6482-6486.

[7] G.L. Newton, K. Arnold, M.S. Price, C. Sherrill, S.B. Delcardayre, Y. Aharonowitz, G. Cohen, J. Davies, R.C. Fahey, C. Davis, Distribution of thiols in microorganisms: mycothiol is a major thiol in most actinomycetes, J. Bacteriol. 178 (1996) 1990-1995.

[8] J.F. Collet, J. Messens, Structure, function, and mechanism of thioredoxin proteins, Antioxid. Redox Signal. 13 (2010) 1205-1216.

[9] W.A. Prinz, F. Åslund, A. Holmgren, J. Beckwith, The role of the Thioredoxin and Glutaredoxin pathways in reducing protein disulfide bonds in the Escherichia coli cytoplasm, J. Biol. Chem. 272 (1997) 15661-15667.

[10] C.H. Lillig, C. Berndt, Glutaredoxins in thiol/disulfide exchange, Antioxid. Redox Signal. 18 (2013) 1654-1665.

[11] J.L. Martin, Thioredoxin-a fold for all reasons, Structure 3 (1995) 245-250.

[12] A. Holmgren, Thioredoxin, Annu. Rev. Biochem. 54 (1985) 237-271.

[13] A. Holmgren, Enzymatic reduction-oxidation of protein disulfides by thioredoxin, Methods Enzymol. 107 (1984) 295-300.

[14] G. Hall, T.D. Bradshaw, C.A. Laughton, M.F. Stevens, J. Emsley, Structure of Mycobacterium tuberculosis thioredoxin in complex with quinol inhibitor PMX464, Protein Sci. 20 (2011) 210-215.

[15] L.S. Jones, B. Yazzie, C.R. Middaugh, Polyanions and the proteome, Mol. Cell. Proteomics 3 (2004) 746-769.

16] J.B. Harborne, C.A. Williams, Advances in flavonoid research since 1992, Phytochemistry 55 (6) (2000) 481-504.

[17] P.G. Pietta, Flavonoids as antioxidants, J. Nat. Prod. 63 (7) (2000) 1035-1042.

[18] M.K. Anwer, R. Al-Shdefat, S. Jamil, P. Alam, M.S. Abdel-Kader, F. Shakeel, Solubility of bioactive compound hesperidin in six pure solvents at (298.15 to 333.15 ) K, J. Chem. Eng. Data 59 (6) (2014) 2065-2069.

[19] S. Majumdar, R. Srirangam, Solubility, stability, physicochemical characteristics and in vitro ocular tissue permeability of hesperidin: a natural bioflavonoid, Pharm. Res. 26 (5) (2009) 1217-1225.

[20] G.R. Grimsley, C.N. Pace, Spectrophotometric determination of protein concentration, Curr. Protoc. Protein Sci. 3 (1) (2004) 1-3.1.9.

[21] N. Sreerama, R.W. Woody, Computation and analysis of protein circular dichroism spectra, Methods Enzymol. 383 (2004) 318-351.

[22] M. Bieri, A.H. Kwan, M. Mobli, G.F. King, J.P. Mackay, P.R. Gooley, Macromolecular NMR spectroscopy for the non-spectroscopist: beyond macromolecular solution structure determination, FEBS J. 278 (2011) 704-715.

[23] S. Rahlfs, M. Fischer, K. Becker, Plasmodium falciparum possesses a classical glutaredoxin and a second, glutaredoxin-like protein with a PICOT homology domain, J. Biol. Chem. 276 (2001) 37133-37140.

[24] H. Kurooka, K. Kato, S. Minoguchi, Y. Takahashi, J.E. Ikeda, S. Habu, N. Osawa, A.M. Buchberg, K. Moriwaki, H. Shisa, T. Honjo, Cloning and characterization of the nucleoredoxin gene that encodes a novel nuclear protein related to thioredoxin, $\mathrm{Ge}-$ nomics 39 (1997) 331-339.

[25] A. Jordan, F. Åslund, E. Pontis, P. Reichard, A. Holmgren, Characterization of Escherichia coli Nrdh a glutaredoxin-like protein with a thioredoxin-like activity profile, J. Biol. Chem. 272 (1997) 18044-18050.

[26] A. Holmgren, Glutathione-dependent synthesis of deoxyribonucleotides. Characterization of the enzymatic mechanism of Escherichia coli glutaredoxin, J. Biol. Chem. 254 (1979) 3672-3678.

[27] M. J. Frisch, G.W. Trucks, H.B. Schlegel, G.E. Scuseria, M.A. Robb, J.R. Cheeseman, G. Scalmani, V. Barone, B. Mennucci, G.A. Petersson, H. Nakatsuji, M. Caricato, X. Li, H.P. Hratchian, A.F. Izmaylov, J. Bloino, G. Zheng, D.J. Sonnenb, Gaussian 09, Revision C.01, http://gaussian.com/

[28] J. Wang, W. Wang, P.A. Kollman, D.A. Case, Automatic atom type and bond type perception in molecular mechanical calculations, J. Mol. Graph. Model. 25 (2006) 247-260.

[29] J.M. Wang, R.M. Wolf, J.W. Caldwell, P. Kollman, D. Case, Development and testing of a general amber force field, J. Comput. Chem. 25 (2004) 1157-1174.

[30] O. Trott, A.J. Olson, AutoDock Vina: improving the speed and accuracy of docking with a new scoring function, efficient optimization, and multithreading, J. Comput. Chem. 31 (2009) (NA-NA).

[31] M.F. Sanner, Python: a programming language for software integration and development, J. Mol. Graph. Model. 17 (1999) 57-61.

[32] D.A. Case, D.S. Cerutti, T.E. Cheatham III, T.A. Darden, R.E. Duke, T.J. Giese, H. Gohlke A.W. Goetz, D. Greene, N. Homeyer, S. Izadi, A. Kovalenko, T.S. Lee, S. LeGrand, P. Li, C. Lin, J. Liu, T. Luchko, R. Luo, D. Mermelstein, K.M. Merz, G. Monard, H., D. M. Y. and P. A. K, AMBER, 2017.

[33] J.A. Maier, C. Martinez, K. Kasavajhala, L. Wickstrom, K.E. Hauser, C. Simmerling, ff14SB: improving the accuracy of protein side chain and backbone parameters from ff99SB, J. Chem. Theory Comput. 11 (2015) 3696-3713.

[34] J.C. Gordon, J.B. Myers, T. Folta, V. Shoja, L.S. Heath, A. Onufriev, H++: a server for estimating pKas and adding missing hydrogens to macromolecules, Nucleic Acids Res. 33 (2005) 368-371.

[35] E. Socher, H. Sticht, Mimicking titration experiments with MD simulations: a protocol for the investigation of pH-dependent effects on proteins, Sci. Rep. 6 (2016), 22523.

[36] D.R. Roe, T.E. Cheatham III, PTRAJ and CPPTRAJ: software for processing and analysis of molecular dynamics trajectory data, J. Chem. Theory Comput. 9 (2013) 3084-3095.

[37] R.A. Laskowski, Enhancing the functional annotation of PDB structures in PDBsum using key figures extracted from the literature, Bioinformatics 23 (2007) 1824-1827. 
[38] L. Tasic, B. Mandic, C.H.N. Barros, D.Z. Cypriano, D. Stanisic, L.G. Schultz, L. da Silva, M.A.M. Mariño, V.L. Queiroz, Exploring bioactivity of hesperidin, naturally occurring flavanone glycoside, isolated from oranges, in: D. Simmons (Ed.), Citrus Fruits, Nova Science Publishers, Inc., ISBN: 978-1-63484-079-8, 2016.

[39] D. Kosek, S. Kylarova, K. Psenakova, L. Rezabkova, P. Herman, J. Vecer, V. Obsilova, T. Obsil, Biophysical and structural characterization of the thioredoxin-binding domain of protein kinase ASK1 and its interaction with reduced thioredoxin, J. Biol. Chem. 289 (2014) 24463-24474

[40] G. Roos, N. Foloppe, K. Van Laer, L. Wyns, L. Nilsson, P. Geerlings, J. Messens, How thioredoxin dissociates its mixed disulfide, PLoS Comput. Biol. 5 (2009), e1000461.

[41] M. Rawat, Y. Av-Gay, Mycothiol-dependent proteins in actinomycetes, FEMS Microbiol. Rev. 31 (2007) 278-292.

[42] I.K. Cheah, B. Halliwell, Ergothioneine; antioxidant potential, physiological function and role in disease, BBA-Mol. Basis Dis. 1822 (2012) 784-793.

[43] R.P. McGeary, A.J. Bennett, Q.B. Tran, K.L. Cosgrove, B.P. Ross, Suramin: clinical uses and structure-activity relationships, Mini Rev. Med. Chem. 8 (2008) 1384-1394.

[44] I. Capila, R.J. Linhardt, Heparin-protein interactions, Angew. Chem. Int. Ed. Engl. 41 (2002) 390-412.

[45] O. Benavente-Garcia, J. Castillo, Update on uses and properties of citrus flavonoids: new findings in anticancer, cardiovascular, and anti-inflammatory activity, J. Agric. Food Chem. 56 (2008) 6185-6205.

[46] J.A. Manthey, N. Guthrie, K. Grohmann, Biological properties of citrus flavonoids pertaining to cancer and inflammation, Curr. Med. Chem. 8 (2001) 135-153.

[47] J. Yu, L. Wang, R.L. Walzem, E.G. Miller, L.M. Pike, B.S. Patil, Antioxidant activity of citrus limonoids, flavonoids, and coumarins, J. Agric. Food Chem. 53 (2005) 2009-2014.

[48] A. Garg, S. Garg, L.J.D. Zaneveld, A.K. Singla, Chemistry and pharmacology of the citrus bioflavonoid hesperidin, Phytother. Res. 15 (2001) 655-669.
[49] R.J. Eberle, L.A. Kawai, F. R de Moraes, L Tasic, R.K Arni, M.A. Coronado, Biochemical and biophysical characterization of a mycoredoxin protein glutaredoxin A1 from Corynebacterium pseudotuberculosis, Int. J. Biol. Macromol. 8130 (2017) (32733-2)

[50] S.L. Hwang, P.H. Shih, G.C. Yen, Neuroprotective effects of citrus flavonoids, J. Agric. Food Chem. 60 (2012) 877-885.

[51] W.D. Comper (Ed.), Heparin and Related Polysaccharides, D.A. Lane, I. Björk, U. Lindahl (Eds.),Vol. 7, Springer Science \& Business Media, Berlin, 1981.

[52] T A Brown, T.M. Yang T. Zaitsevskaia, Y. Xia, C.A. Dunn, R.O. Sigle, B. Knudsen, W.G. Carter, Adhesion or plasmin regulates tyrosine phosphorylation of a novel membrane glycoprotein p80/gp140/CUB domain-containing protein 1 in epithelia, J. Biol. Chem. 279 (2004) 14772-14783.

[53] F. Manetti, F. Corelli, M. Botta, Fibroblast growth factors and their inhibitors, Curr. Pharm. Des. 6 (2000) 1897-1924.

[54] J. Chartron, C. Shiau, C.D. Stout, K.S. Carroll, 3'-Phosphoadenosine-5'-phosphosulfate reductase in complex with thioredoxin: a structural snapshot in the catalytic cycle, Biochemistry 46 (2007) 3942-3951.

[55] J. Qin, G.M. Clore, W.P. Kennedy, J.R. Huth, A.M. Gronenborn, Solution structure of human thioredoxin in a mixed disulfide intermediate complex with its target peptide from the transcription factor NFkB, Structure 3 (1995) 289-297.

[56] B.W. Lennon, C.H. Williams, M.L. Ludwig, Twists in catalysis: alternating conformations of Escherichia coli thioredoxin reductase, Science 289 (2000) 1190-1194.

[57] Y. Li, Y. Hu, X. Zhang, H. Xu, E. Lescop, B. Xia, C. Jin, Conformational fluctuations coupled to the thiol-disulfide transfer between thioredoxin and arsenate reductase in Bacillus subtilis, J. Biol. Chem. 282 (2007) 11078-11083.

[58] J. Chartron, C. Shiau, C.D. Stout, K.S. Carroll, 3'-Phosphoadenosine-5'-phosphosulfate reductase in complex with thioredoxin: a structural snapshot in the catalytic cycle, Biochemistry 46 (2007) 3942-3951. 